\title{
Review Article \\ Effectiveness of Palivizumab in Preventing RSV Hospitalization in High Risk Children: A Real-World Perspective
}

\author{
Nusrat Homaira, ${ }^{1}$ William Rawlinson, ${ }^{2,3,4}$ Thomas L. Snelling, ${ }^{5}$ and Adam Jaffe ${ }^{1}$ \\ ${ }^{1}$ Disciplines of Paediatrics, School of Women's and Children's Health, UNSW, Sydney Children's Hospital, Level 3, \\ Emergency Wing, Randwick, Sydney, NSW 2031, Australia \\ ${ }^{2}$ Virology Division, SEALS Microbiology, Prince of Wales Hospital, Randwick, Sydney, NSW 2031, Australia \\ ${ }^{3}$ School of Medical Sciences, UNSW, Sydney, NSW, Australia \\ ${ }^{4}$ Biotechnology and Biomolecular Sciences, UNSW, Sydney, NSW, Australia \\ ${ }^{5}$ Telethon Kids Institute, University of Western Australia, 100 Roberts Road, Subiaco, WA 6009, Australia \\ Correspondence should be addressed to Nusrat Homaira; z3442406@student.unsw.edu.au
}

Received 1 September 2014; Revised 15 October 2014; Accepted 15 October 2014; Published 4 December 2014

Academic Editor: Hans Juergen Laws

Copyright (C) 2014 Nusrat Homaira et al. This is an open access article distributed under the Creative Commons Attribution License, which permits unrestricted use, distribution, and reproduction in any medium, provided the original work is properly cited.

\begin{abstract}
Infection with respiratory syncytial virus (RSV) is one of the major causes globally of childhood respiratory morbidity and hospitalization. Palivizumab, a humanized monoclonal antibody, has been recommended for high risk infants to prevent severe RSV-associated respiratory illness. This recommendation is based on evidence of efficacy when used under clinical trial conditions. However the real-world effectiveness of palivizumab outside of clinical trials among different patient populations is not well established. We performed a systematic review focusing on postlicensure observational studies of the protective effect of palivizumab prophylaxis for reducing RSV-associated hospitalizations in infants and children at high risk of severe infection. We searched studies published in English between 1 January 1999 and August 2013 and identified 420 articles, of which 20 met the inclusion criteria. This review supports the recommended use of palivizumab for reducing RSV-associated hospitalization rates in premature infants born at gestational age $<33$ weeks and in children with chronic lung and heart diseases. Data are limited to allow commenting on the protective effect of palivizumab among other high risk children, including those with Down syndrome, cystic fibrosis, and haematological malignancy, indicating further research is warranted in these groups.
\end{abstract}

\section{Introduction}

Globally, respiratory syncytial virus (RSV) is one of the major causes of childhood acute lower respiratory infection (ALRI) associated morbidity, hospitalization, and mortality. In 2005, there were an estimated 3.4 (95\% CI 2.8-4.3) million episodes of severe RSV-associated childhood ALRIs necessitating hospital admission [1]. The risk of RSV infection is highest in children born prematurely or those with existing comorbidities including bronchopulmonary dysplasia/ chronic lung disease (BPD/CLD), congenital heart disease (CHD), cystic fibrosis (CF), multiple congenital anomalies, and immunodeficiency [2-4].

The reported RSV hospitalization rate in premature infants ranges between 5.2\% and 16.8\% [5-8]. RSV hospitalization of high risk infants and children is associated with significant health care resource utilization and monetary costs $[9,10]$. A high proportion of high risk infants and children hospitalized with RSV infection require admission to an intensive care unit and mechanical ventilation [11]. In the United States it has been estimated that the annual cost of hospitalization for RSV pneumonia in children aged $<4$ years is approximately $\$$ US3 to 4 million [12]. There are additional costs associated with outpatient visits, follow-up, and lost productivity due to parents taking time off work to care for sick children [10].

These factors make prevention of RSV infection a global public health priority, although no active vaccination strategy is yet available. Palivizumab, a humanized monoclonal antiRSV antibody, was shown to reduce hospitalizations and the clinical severity of RSV infection in high risk infants and children by $55 \%$ in a randomised controlled trial [3]. The updated 
recommendation of the American Academy of Pediatrics (AAP) recommends prophylactic use of palivizumab for children with chronic lung disease of prematurity, congenital heart disease, or other chronic illnesses and for children born at gestational age (GA) less than 29 weeks [13]. The AAP recommends that eligible children should receive no more than five monthly doses of palivizumab [13]. Though guidelines for use of palivizumab for children born with chronic illness are similar across the developed countries, the cut-off gestational age for premature infants varies widely. New Zealand's recommendation is for use in premature infants born at GA $\leq 28$ weeks, infants discharged home on oxygen, or those born at GA 29-32 weeks with birth weight of $1000 \mathrm{~g}$ or less [14]. The cut-off for GA is $<26$ weeks in Sweden [15]. Recommendations from Australia and Canada include children born up to 32-35 weeks with two or more risk factors associated with RSV infection $[16,17]$. Currently, palivizumab is not routinely recommended for use in children with immune deficiencies, Down syndrome, cystic fibrosis, upper airway obstruction, or other chronic lung diseases.

The cost of a single course of palivizumab is estimated to be as much as \$ US4458 per child [18]. Due to its high cost and limited evidence of cost-effectiveness [19], the use of palivizumab is restricted even in well-resourced settings. The cost-effectiveness of palivizumab is largely dependent on its effectiveness across specific subgroups of high risk children.

Several systematic reviews have demonstrated the effectiveness of palivizumab in reducing the subsequent hospitalization rates in high risk children. These reviews have concluded that palivizumab is effective in reducing RSV hospitalization rates by $>40 \%$ in premature infants and high risk children. However most of these reviews have included efficacy trials conducted under strict controlled environment [20-22]. While randomised trials provide high level evidence of the efficacy of an intervention under idealised conditions, postlicensure observational studies assess the "real-world" value of an intervention under more realistic conditions, where eligibility criteria are less rigidly applied and doses may be delayed or omitted [23]. The objective of this narrative systematic review was to identify which high risk infants and children have been proven to benefit most from palivizumab prophylaxis under real-world practice settings, to identify gaps in existing knowledge, and to provide recommendations for future research.

\section{Methods}

\subsection{Selection Criteria for the Studies}

2.1.1. Study Type. Only full-length peer-reviewed observational studies (cohort, case-control, and cross-sectional studies including survey) published in English were included in the review. As the first randomised trial was published in September 1998 [3], we included studies that were published between January 1999 and August 2013. We excluded conference proceedings, review articles, or editorial reports. We also excluded clinical trial data as we were interested only in the "real-world" effectiveness of palivizumab.
2.1.2. Study Participants. Study participants were high risk infants and children aged $<2$ years, including premature infants (GA $<37$ weeks) and children with any chronic congenital conditions that may put them at risk of severe RSV disease.

2.1.3. Outcome Measure. As the primary interest for this review was the incidence/rate of RSV hospitalization as a measure of prevention of severe RSV disease by immunoprophylaxis with palivizumab, only studies that examined the effect of palivizumab on subsequent RSV hospitalization were included.

2.2. Identification of the Studies. We conducted a comprehensive MEDLINE search using the MESH terms "respiratory syncytial virus and primary prevention/immunization," "respiratory syncytial virus and secondary prevention," "respiratory syncytial virus and antibodies, monoclonal/or antibodies, monoclonal, humanized/," "respiratory syncytial virus and hospitalization," "respiratory syncytial virus and antiviral," "respiratory syncytial virus and passive immunization," "respiratory syncytial virus and palivizumab," and "respiratory syncytial virus and child." Secondary searches were performed using EMBASE, CINHAL, and Global Health databases using the same keywords. Additional literature was identified by searching the citation list of the identified articles. We also looked for relevant literature using Google Scholar. All the searched results were merged into one single document and all duplicates were removed using Endnote. Once duplicates were removed, we examined the title and the abstract of the literature to exclude articles that did not meet our inclusion criteria. The full length of the relevant articles was retrieved and examined to further determine if they met inclusion criteria.

Each full-length article was reviewed by one researcher $(\mathrm{NH})$ and the summary information extracted using a standardised form following the PRISMA guideline [24]. The data extracted for each article were author, year, and country of publication, time frame of the study, study population, sample size, intervention strategy, dosing regimen, study outcomes, and conclusion. The investigator then shared the abstracted summary form with all other investigators for their review and consensus.

\section{Results}

The initial search identified 420 articles (Figure 1). After removing studies that did not meet our inclusion criteria, 20 studies were included: 12 were cohort studies, 7 were record reviews, and one was a questionnaire-based survey. The included studies were conducted in the USA, Canada, Australia, Japan, Korea, and Europe (Figure 2).

\subsection{Effectiveness of Palivizumab for Preventing RSV-Associated} Hospitalization in Premature Infants (Table 1). Twelve papers $[23,25-35]$ reported the effectiveness of palivizumab in reducing RSV hospitalization in 89,469 premature infants with or without CLD. Five studies included premature infants 


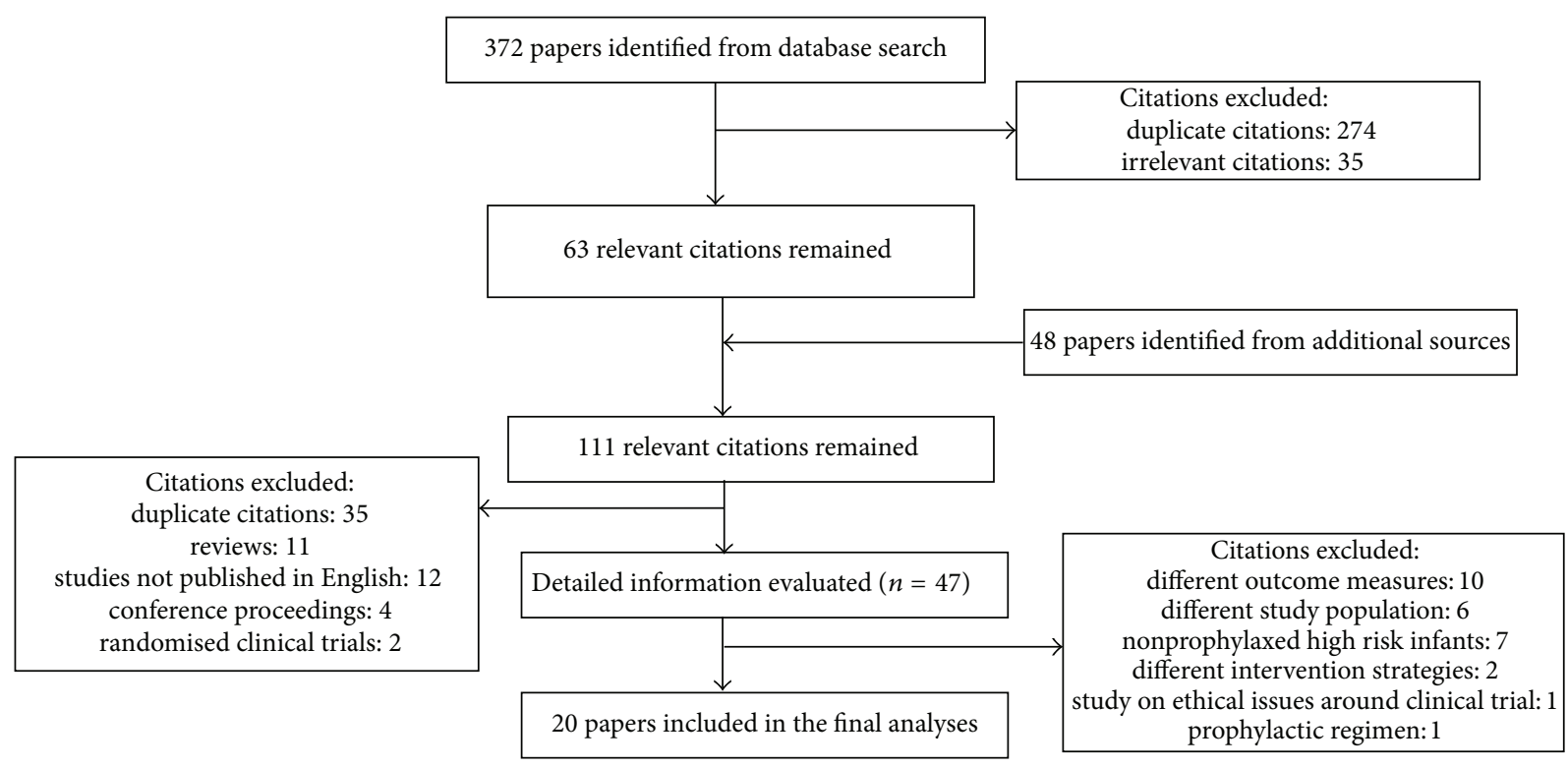

FIGURE 1: Flow diagram for selection of papers for the review.

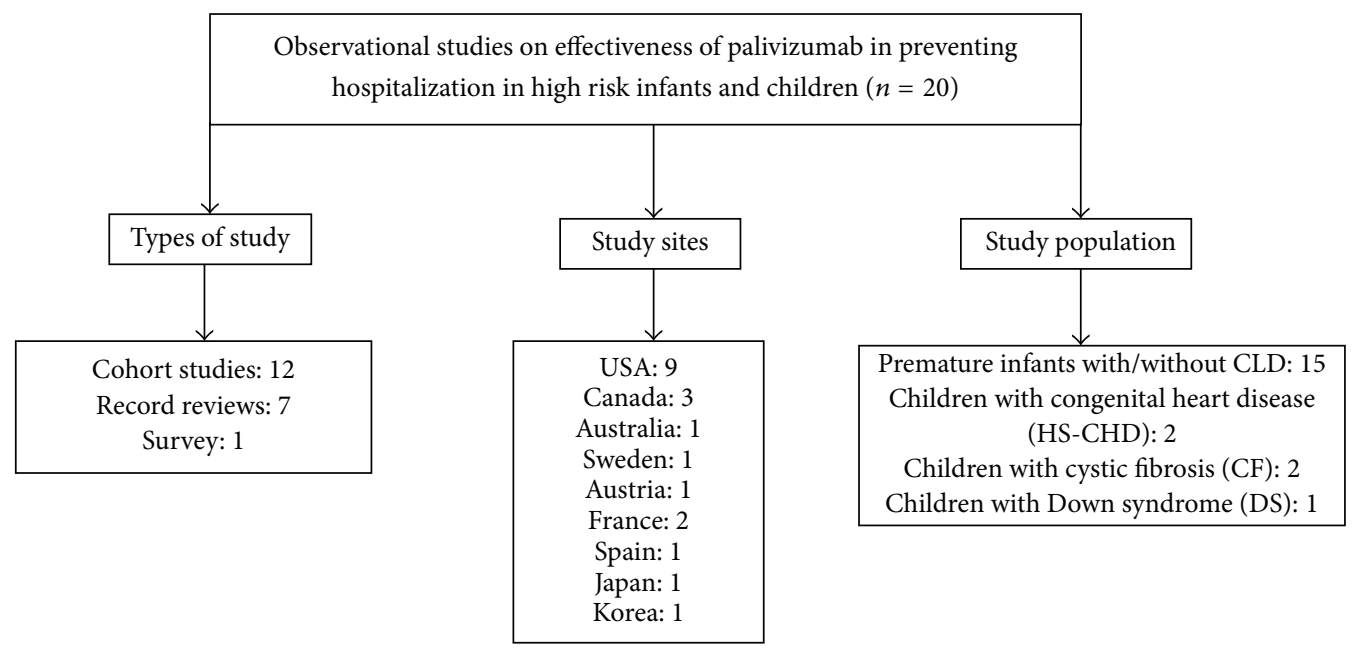

FIGURE 2: Characteristics of the selected studies on effectiveness of palivizumab in reducing RSV-associated hospitalization in high risk infants and children.

born at GA < 36 weeks with or without CLD [23, 26, 28, $34,35]$, three studies included only very preterm infants born at GA < 33 weeks [29-31], and four included only premature infants with CLD $[23,25,30,34,36]$. Two included studies compared differences in the rate of RSV hospitalization among prophylaxed and nonprophylaxed infants over one RSV season $[25,29]$, five studies compared rates in prophylaxed and nonprophylaxed groups over noncontemporaneous seasons [23, 26-28, 31], and in two studies rates in prophylaxed infants were compared with rates in nonprophylaxed infants from other published studies [33, 34].

Two of the five studies $[26,28]$ which included any premature infant born at $\mathrm{GA}<36$ weeks reported a statistically significant reduction $(P<0.05)$ in RSV hospitalization among prophylaxed compared to nonprophylaxed infants (19-29\% rate reduction). Two of the remaining three studies $[33,34]$ did not have a contemporaneous comparison group or compared the rates to children from other published studies $[3,8]$. The remaining study [23] which included late preterm infants (GA 33 to 35 weeks) reported a nonsignificant reduction of $0.6 \%$. Some of the significant risk factors associated with increased risk of RSV hospitalization in premature infants born at GA $\leq 35$ weeks included preterm births at $\mathrm{GA}<31$ weeks, intrauterine growth retardation, single mother family [29], and having siblings at home, more than 5 individuals in the household, and smokers at home [35].

In all of the identified studies which included infants born at GA $<32$ weeks, the rate of RSV hospitalization was lower in infants who received palivizumab [27, 29-31, 34]; 


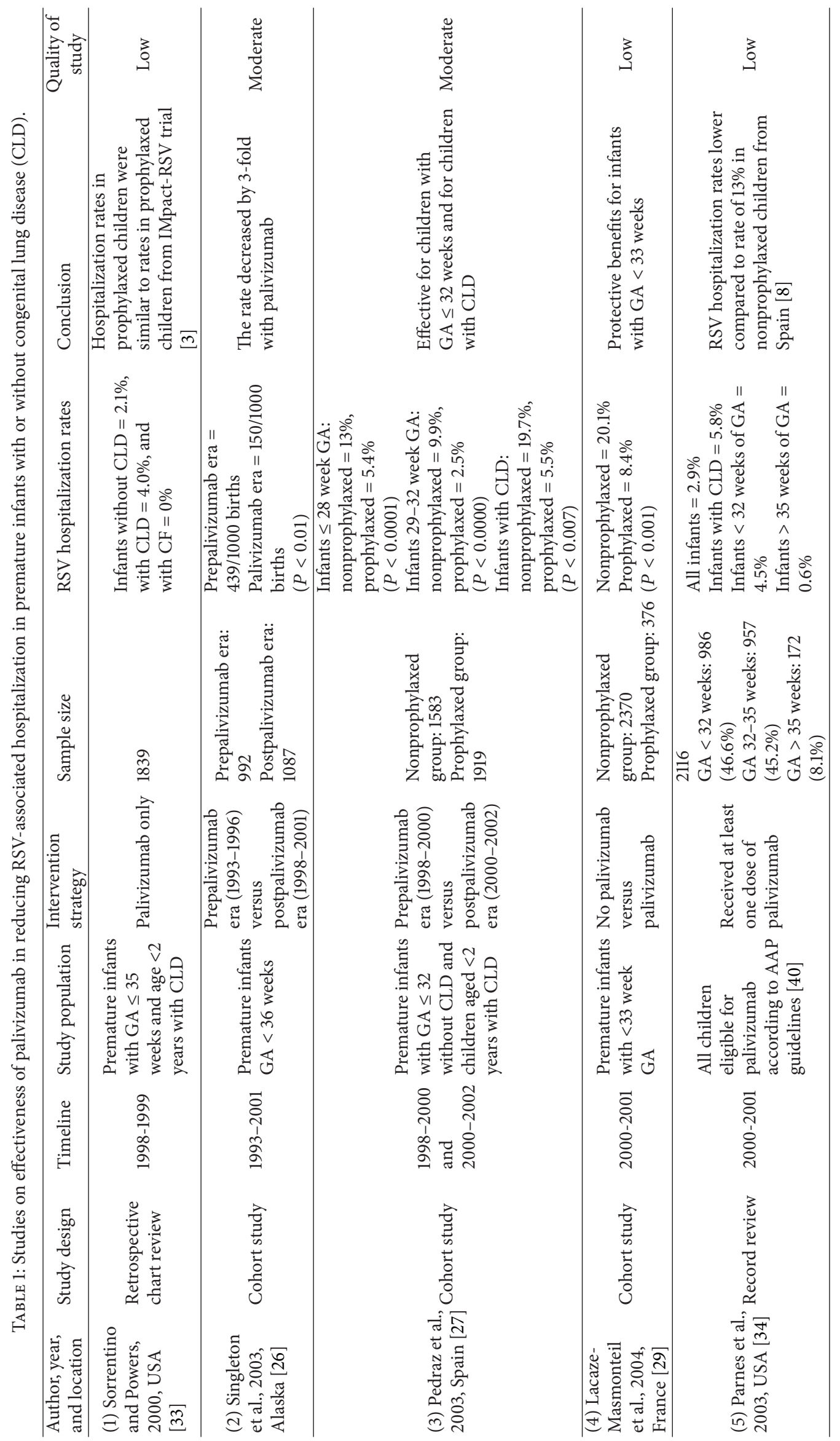




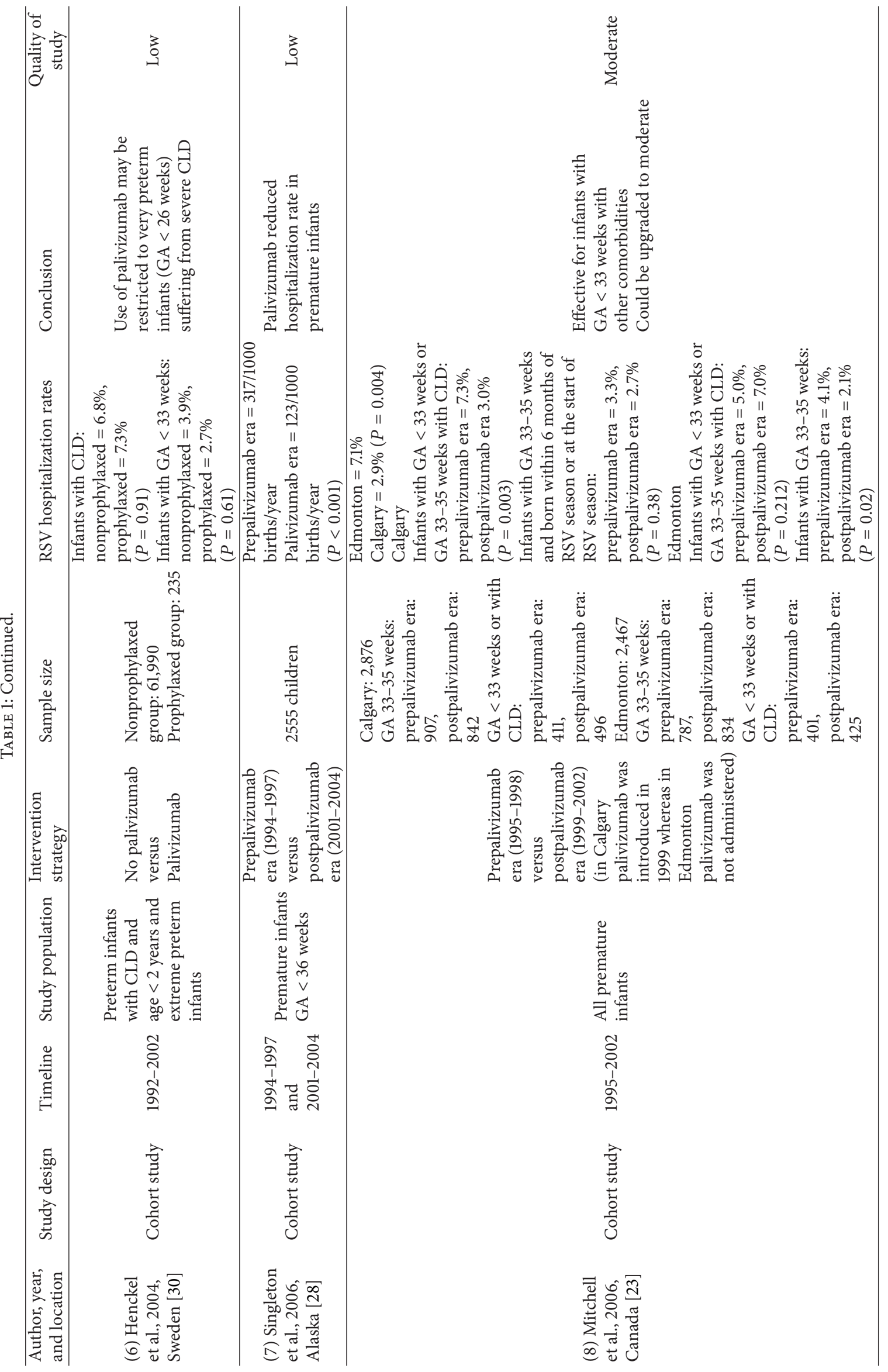




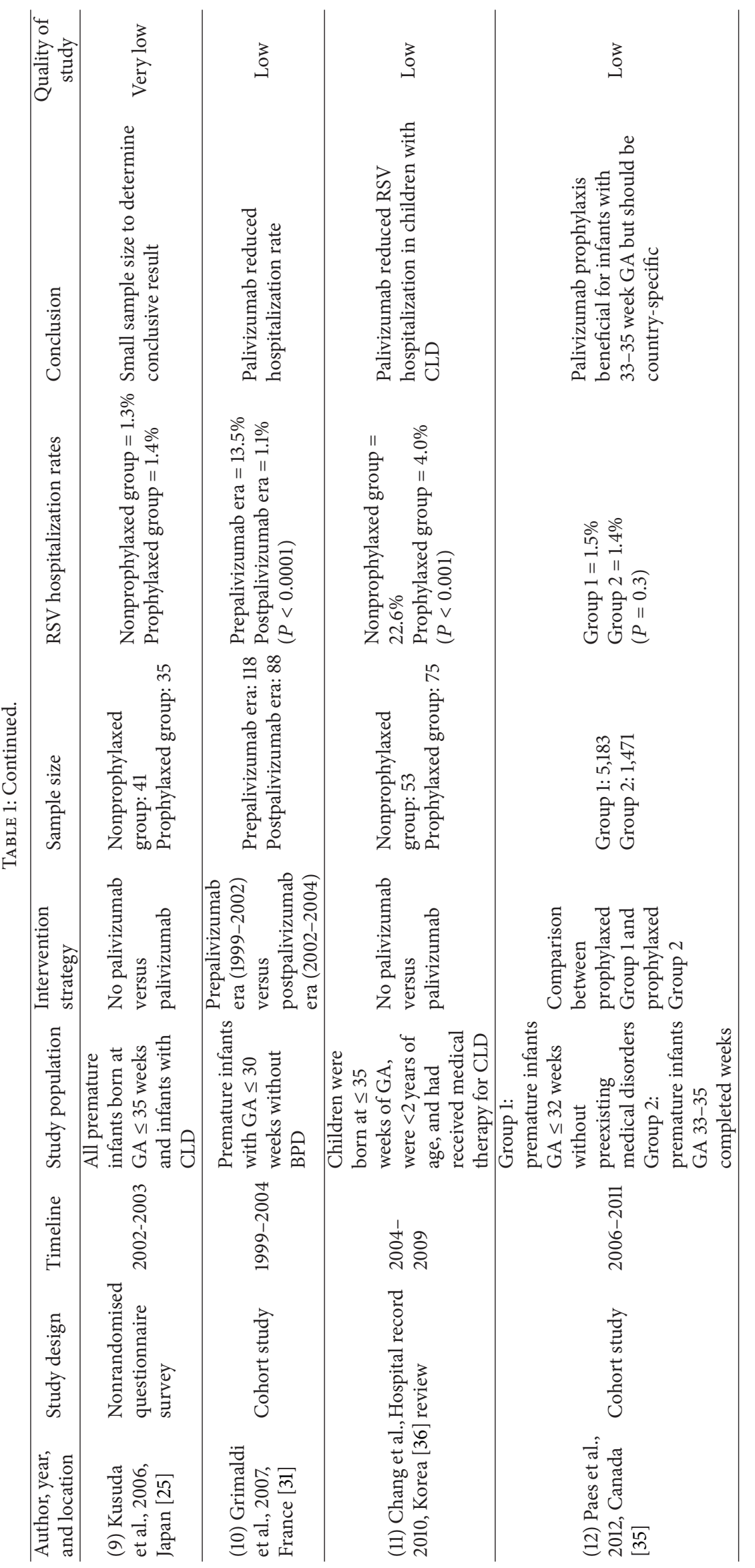


the reported reduction ranged between $1.2 \%$ and $12.4 \%$ and in 4 of 5 studies $[23,27,29,31]$ this reduction was statistically significant. Seven of eight studies [23, 25-27, 30, 33, 34, 36] that included premature infants with chronic lung disease reported a statistically significant reduction in children who received palivizumab compared to those who did not; the reduction varied between 0.5 and $29 \%$.

3.2. Effectiveness of Palivizumab in Preventing RSV-Associated Hospitalization in Children with Hemodynamically Significant Congenital Heart Disease (HS-CHD) (Table 2). One study based on hospital record review [37] investigated the effect of palivizumab among 266 children aged $<2$ years with $\mathrm{CHD}$ (Table 2). Rates of RSV hospitalization in prophylaxed children were $19 \%$ lower than for nonprophylaxed infants from a different RSV season. The authors concluded the reduction was modest compared to the effect demonstrated in the multinational trial that investigated palivizumab in children with CHD [38] and recommended further assessment of the real-world benefit of palivizumab in this group of children.

3.3. Effectiveness of Palivizumab in Preventing RSV-Associated Hospitalization in Children with Cystic Fibrosis (CF) (Table 2). Two studies included 2966 children with cystic fibrosis [39, 40]. One study compared RSV-associated hospitalization rates between prophylaxed and nonprophylaxed children during the same RSV season, reporting a nonsignificant reduction of $0.2 \%$ in the prophylaxed group. A retrospective record review of children with CF listed on a hospital based palivizumab registry reported significantly lower odds of RSV hospitalization in prophylaxed children compared to the reported risk from other published studies.

\subsection{Effectiveness of Palivizumab in Preventing RSV-Associated} Hospitalization in Children with Down Syndrome (Table 2). One study used data from the Canadian Registry of Palivizumab to compare the rate of RSV hospitalization among 600 children with Down syndrome who received prophylaxis with that of 12,710 children without Down syndrome who received palivizumab [41]. The hospitalization rate in children with Down syndrome $(1.53 \%)$ was similar to that of other children receiving palivizumab (1.45\%).

3.5. Association between Dosing of Palivizumab and RSVAssociated Hospitalization (Table 3). The number of Palivizumab doses per child in each RSV season varied between the included studies. Three studies [34, 37, 42, 43] reported an association between the number of doses of palivizumab and the subsequent RSV-associated hospitalization rate in 4,372 children. Palivizumab registry review showed that missed or delayed dosing of palivizumab was associated with statistically significant increase in hospitalization rate in infants who missed or delayed a dose compared to those who were compliant with the scheduled dosing regimen of palivizumab (4.4\% versus $2.4 \% ; P=0.02$ ) [34]. A cohort study [43] that included premature infants of GA 29 to 32 weeks also reported a lower RSV hospitalization rate in children prophylaxed according to the standard recommendation of
5 doses, compared with the rate in children who received inadequate prophylaxis, that is, children who did not receive the recommended five full doses (3.3\% versus $8.1 \%, P=$ 0.07). Another cohort study [37] among children with HS$\mathrm{CHD}$ reported a reduction in RSV hospitalizations from 7-9 cases/year when children received palivizumab ad hoc to 2-3 cases/per year when prophylaxis was administered systematically and in accordance with recommendations $(P=0.03)$. One study [44] reported a lower incidence of RSV hospitalization among 17,641 children who received palivizumab at home $(0.4 \%)$ compared to 1226 children who received it in a clinic $(1.2 \%)(P=0.014)$.

\section{Methodological Quality of the Studies}

We used the Cochrane GRADE approach to rate the quality of the included studies [45]. The GRADE approach is probably of most relevance for clinical trials as observational studies are inherently prone to bias. However observational studies can nonetheless be upgraded or downgraded based on design, consistency, and precision of results, directness of the evidence, risk of bias, and presence of confounders [45]. Two of the studies included in this review were downgraded to a "very low" quality due to small sample size [25] and lack of a contemporary comparison group [39] limiting the external validity of the study findings. Due to directness of evidence, precision, and comparability of the results, three of the included studies were upgraded to a moderate quality $[23,26,27]$ while no other studies required downgrading.

\section{Discussion}

Palivizumab prophylaxis was first shown to reduce RSVassociated hospitalization by $39-78 \%$ in premature infants and in children with CLD/BPD in a multinational randomized controlled trial [3]. This landmark trial was subsequently followed by several postlicensure studies in various settings which were included in this review. Our review of these studies found that they also support protective effect of palivizumab against RSV hospitalization among premature infants and in children with CLD, although the size of benefit across the studies was more modest than reported in the clinical trial. Although we did identify studies that reported statistically significant reduction of RSV hospitalization rates among all premature infants with $\mathrm{GA}<36$ weeks $[26,28]$, these studies did not stratify the effectiveness in children born at GA 32-35 weeks with additional risk factors. This makes the results inconclusive for premature infants born at GA 3235 weeks. The only study [23] that stratified by gestational age did not find a significant reduction in hospitalization rate in prophylaxed infants born at GA 33 to 35 weeks without CLD. The review of the studies suggests that groups of children who are likely to benefit most from palivizumab prophylaxis are premature infants born at GA $\leq 32$ weeks and children with CLD. Though some international guidelines recommend use of palivizumab in premature infants born at GA $<29$ weeks [13-15], highly resourced countries may also 


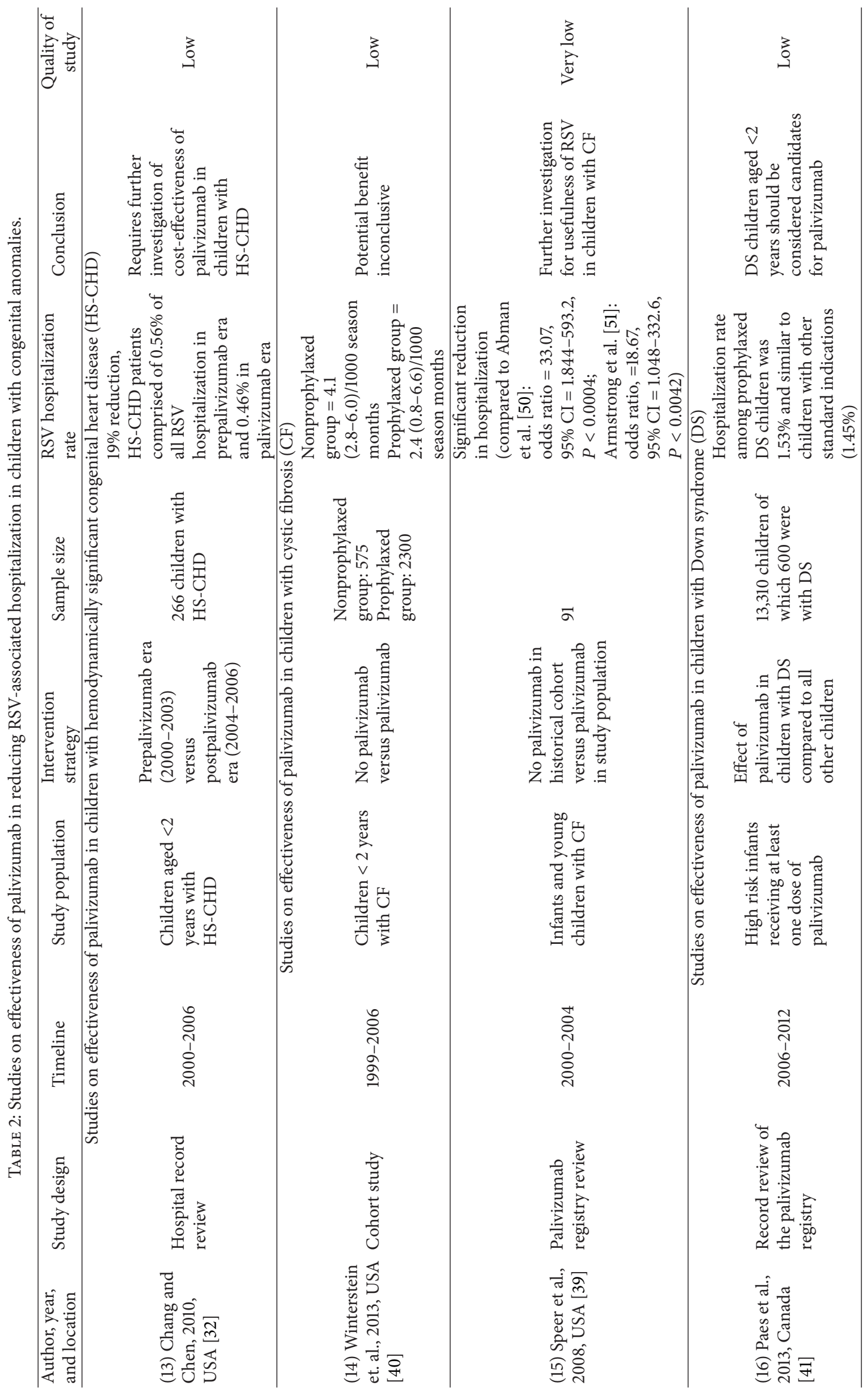




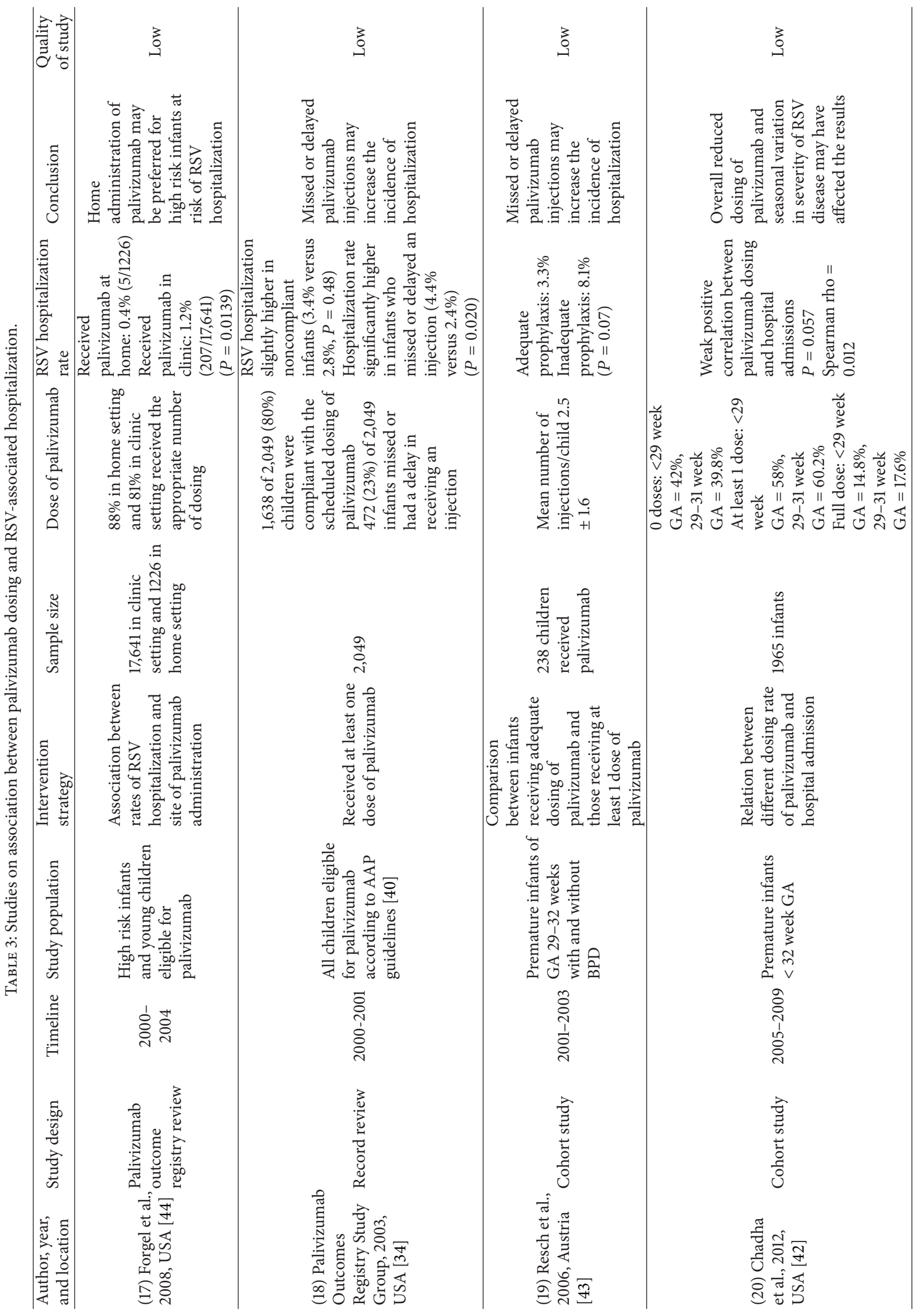




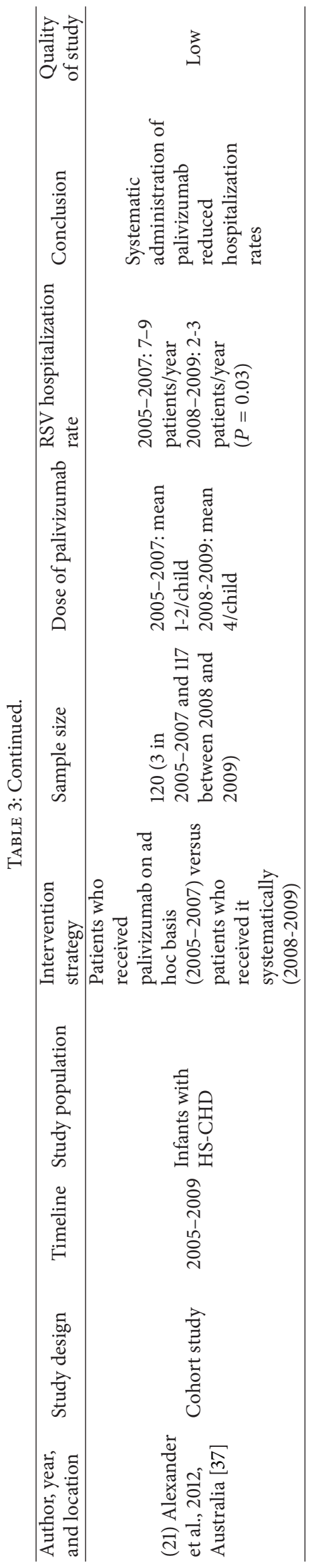


consider palivizumab for premature infants born at GA 2932 weeks as per Canadian and Australian recommendations $[16,17]$.

One study found a lower rate of RSV hospitalization among children with HS-CHD who received palivizumab [32], a group widely recommended for prophylactic use of palivizumab in international guidelines [13-17]. This reduction, however, was not statistically significant and was much less than the clinical trial that demonstrated a reduction of $45 \%$ in children with HS-CHD [38]. The low observed reduction may be attributed to the fact that the study groups were not contemporaneous and RSV transmission may vary across seasons. It may also be that organised and systematic administration of palivizumab is required to achieve a benefit in children with CHD [46]. We identified only one observational study of children with HS-CHD which limits our ability to draw firm conclusion for this group. However as all the guidelines have prioritised children with HS-CHD it may be beneficial to consider these children for five monthly doses of palivizumab during the first RSV season [13].

Three studies [39-41] examined the effect of palivizumab immunoprophylaxis for preventing RSV-associated hospitalization in other groups of high risk children who are not covered by specific recommendations in the AAP guidelines [47], such as children with CF and Down syndrome. These studies did not report a statistically significant reduction of RSV hospitalization rate in these groups of children. In the absence of data from well conducted observational studies demonstrating real-world benefit of palivizumab, international guidelines [13-17] which do not recommend routine use of palivizumab in these groups of children seem appropriate.

We endeavoured to identify all potentially relevant observational studies by using a comprehensive search strategy. As with all systematic search strategies it is possible that we have inadvertently overlooked some relevant studies, for example, unpublished work or studies published in languages other than English. Our review was based on observational studies and inability to completely control for confounding factors is a major limitation of the included studies. In particular "confounding by indication," in which infants at highest risk of disease are targeted for prophylaxis, has the potential to result in underestimation of the protective effect. In addition many of the included studies compared rates of hospitalization in children receiving prophylaxis with children not receiving prophylaxis from different cohorts and in different RSV seasons and some were conducted over one RSV season. RSV transmission varies over time and geographical location $[48,49]$ confounding direct interpretation of the results.

We did not include a meta-analysis in our review as the studies included in this review were generally of varying quality, assessed heterogeneous populations, and employed different methodologies limiting quantitative synthesis. The review of the included studies prevents us from drawing firm conclusions except for among preterm infants $(\mathrm{GA}<33$ weeks) and those with CLD and HS-CHD. Further studies of palivizumab in children with Down syndrome, CF, or hematologic malignancies and other high risk groups may inform the current guidelines for immunoprophylaxis. Also studies documenting the association between number of palivizumab injections and RSV infection may better define the optimal dosing of the immunoprophylaxis with respect to burden and cost.

\section{Authors' Conclusion}

RSV continues to be one of the major causes of childhood hospitalization worldwide. This review supports use of palivizumab for premature infants born at gestational age $<33$ weeks and in selected subgroups of children at high risk of developing severe RSV disease, in particular children with CLD or HS-CHD. Recommendations for targeted immunoprophylaxis for specific groups at high risk for severe RSV disease need to be based on better effectiveness data, along with country-specific data on burden of RSV disease, prevalence of risk factors, and the availability of funds for preventive interventions.

\section{Conflict of Interests}

The authors declare that there is no conflict of interests regarding the publication of this paper.

\section{References}

[1] H. Nair, D. J. Nokes, B. D. Gessner et al., "Global burden of acute lower respiratory infections due to respiratory syncytial virus in young children: a systematic review and meta-analysis," The Lancet, vol. 375, no. 9725, pp. 1545-1555, 2010.

[2] S. A. Madhi, L. Kuwanda, C. Cutland, and K. P. Klugman, "Fiveyear cohort study of hospitalization for respiratory syncytial virus associated lower respiratory tract infection in African children," Journal of Clinical Virology, vol. 36, no. 3, pp. 215-221, 2006.

[3] The IMpact-RSV Study Group, "Palivizumab, a humanized respiratory syncytial virus monoclonal antibody, reduces hospitalization from respiratory syncytial virus infection in high-risk infants," Pediatrics, vol. 102, no. 3, part 1, pp. 531-537, 1998.

[4] E. A. F. Simoes, "Respiratory syncytial virus infection," The Lancet, vol. 354, no. 9181, pp. 847-852, 1999.

[5] T. P. Stevens, R. A. Sinkin, C. B. Hall, W. M. Maniscalco, and K. M. McConnochie, "Respiratory syncytial virus and premature infants born at 32 weeks' gestation or earlier: hospitalization and economic implications of prophylaxis," Archives of Pediatrics and Adolescent Medicine, vol. 154, no. 1, pp. 55-61, 2000.

[6] J. McCormick and R. Tubman, "Readmission with respiratory syncytial virus (RSV) infection among graduates from a Neonatal Intensive Care Unit," Pediatric Pulmonology, vol. 34, no. 4, pp. 262-266, 2002.

[7] J. G. Liese, E. Grill, B. Fischer et al., "Incidence and risk factors of respiratory syncytial virus-related hospitalizations in premature infants in Germany," European Journal of Pediatrics, vol. 162, no. 4, pp. 230-236, 2003.

[8] X. Carbonell-Estrany and J. Quero, "Hospitalization rates for respiratory syncytial virus infection in premature infants born during two consecutive seasons," Pediatric Infectious Disease Journal, vol. 20, no. 9, pp. 874-879, 2001. 
[9] J. S. Sampalis, "Morbidity and mortality after RSV-associated hospitalizations among premature canadian infants," The Journal of Pediatrics, vol. 143, no. 5, pp. 150-156, 2003.

[10] S. Kamal-Bahl, J. Doshi, and J. Campbell, "Economic analyses of respiratory syncytial virus immunoprophylaxis in high-risk infants: a systematic review," Archives of Pediatrics \& Adolescent Medicine, vol. 156, no. 10, pp. 1034-1041, 2002.

[11] E. E. Wang, B. J. Law, and D. Stephens, "Pediatric Investigators Collaborative Network on Infections in Canada (PICNIC) prospective study of risk factors and outcomes in patients hospitalized with respiratory syncytial viral lower respiratory tract infection," The Journal of Pediatrics, vol. 126, no. 2, pp. 212219, 1995.

[12] T. S. Howard, L. H. Hoffman, P. E. Stang, and E. A. F. Simoes, "Respiratory syncytial virus pneumonia in the hospital setting: length of stay, charges, and mortality," The Journal of Pediatrics, vol. 137, no. 2, pp. 227-232, 2000.

[13] Committee on Infectious Diseases and Bronchiolitis Guidelines Committee, "Updated guidance for palivizumab prophylaxis among infants and young children at increased risk of hospitalization for respiratory syncytial virus infection," Pediatrics, vol. 134, p. e620, 2014.

[14] A. M. Vogel, D. R. Lennon, R. Broadbent et al., "Palivizumab prophylaxis of respiratory syncytial virus infection in high-risk infants," Journal of Paediatrics and Child Health, vol. 38, no. 6, pp. 550-554, 2002.

[15] Swedish Consensus Group, "Management of infections caused by respiratory syncytial virus," Scandinavian Journal of Infectious Diseases, vol. 33, no. 5, pp. 323-328, 2001.

[16] G. Ranmuthugala, L. Brown, and B. A. Lidbury, "The burden of disease associated with respiratory syncytial virus in Australia," Discussion Paper 7(64), National Centre for Social and Economic Modelling, Canberra, Australia, 2008.

[17] J. L. Robinson, "Preventing respiratory syncytial virus infections," Paediatrics \& Child Health, vol. 16, no. 8, pp. 488-490, 2011.

[18] S. Kamal-Bahl, J. Doshi, and J. Campbell, "Economic analyses of respiratory syncytial virus immunoprophylaxis in highrisk infants: a systematic review," Archives of Pediatrics and Adolescent Medicine, vol. 156, no. 10, pp. 1034-1041, 2002.

[19] D. Wang, C. Cummins, S. Bayliss, J. Sandercock, and A. Burls, "Immunoprophylaxis against respiratory syncytial virus (RSV) with palivizumab in children: a systematic review and economic evaluation," Health Technology Assessment, vol. 12, no. 36, pp. 186, 2008.

[20] N. D. Embleton, C. Harkensee, and M. C. Mckean, "Palivizumab for preterm infants. Is it worth it?" Archives of Disease in Childhood: Fetal \& Neonatal Edition, vol. 90, no. 4, pp. F286F289, 2005.

[21] E. E. Wang and N. K. Tang, "Immunoglobulin for preventing respiratory syncytial virus infection," Cochrane Database of Systematic Reviews, no. 2, Article ID CD001725, 2000.

[22] J. R. Groothuis and H. Nishida, "Prevention of respiratory syncytial virus infections in high-risk infants by monoclonal antibody (palivizumab)," Pediatrics International, vol. 44, no. 3, pp. 235-241, 2002.

[23] I. Mitchell, S. Tough, L. Gillis, and C. Majaesic, "Beyond randomized controlled trials: a "real life" experience of respiratory syncytial virus infection prevention in infancy with and without palivizumab," Pediatric Pulmonology, vol. 41, no. 12, pp. 11671174, 2006.
[24] D. Moher, A. Liberati, and J. Tetzlaff, "Preferred reporting items for systematic reviews and meta-analyses: the PRISMA statement," Journal of Clinical Epidemiology, vol. 62, no. 10, pp. 10061012, 2009.

[25] S. Kusuda, T. Koizumi, T. Sakai, M. Fujimura, H. Nishida, and H. Togari, "Results of clinical surveillance during the Japanese first palivizumab season in 2002-2003," Pediatrics International, vol. 48, no. 4, pp. 362-368, 2006.

[26] R. Singleton, L. Dooley, D. Bruden, S. Raelson, and J. C. Butler, "Impact of palivizumab prophylaxis on respiratory syncytial virus hospitalizations in high risk Alaska Native infants," The Pediatric Infectious Disease Journal, vol. 22, no. 6, pp. 540-545, 2003.

[27] C. Pedraz, X. Carbonell-Estrany, J. Figueras-Aloy, and J. Quero, "Effect of palivizumab prophylaxis in decreasing respiratory syncytial virus hospitalizations in premature infants," The Pediatric Infectious Disease Journal, vol. 22, no. 9, pp. 823-827, 2003.

[28] R. J. Singleton, D. Bruden, L. R. Bulkow, G. Varney, and J. C. Butler, "Decline in respiratory syncytial virus hospitalizations in a region with high hospitalization rates and prolonged season," The Pediatric Infectious Disease Journal, vol. 25, no. 12, pp. 11161122, 2006.

[29] T. Lacaze-Masmonteil, P. Truffert, D. Pinquier et al., "Lower respiratory tract illness and RSV prophylaxis in very premature infants," Archives of Disease in Childhood, vol. 89, no. 6, pp. 562$567,2004$.

[30] E. Henckel, J. Luthander, E. Berggren et al., "Palivizumab prophylaxis and hospitalization for respiratory syncytial virus disease in the Stockholm infant population, 1999 through 2002," The Pediatric Infectious Disease Journal, vol. 23, no. 1, pp. 27-31, 2004.

[31] M. Grimaldi, B. Gouyon, P. Sagot, C. Quantin, F. Huet, and J. B. Gouyon, "Palivizumab efficacy in preterm infants with gestational age $<$ or $=30$ weeks without bronchopulmonary dysplasia," Pediatric Pulmonology, vol. 42, no. 3, pp. 189-192, 2007.

[32] R.-K. R. Chang and A. Y. Chen, "Impact of palivizumab on RSV hospitalizations for children with hemodynamically significant congenital heart disease," Pediatric Cardiology, vol. 31, no. 1, pp. 90-95, 2010.

[33] M. Sorrentino and T. Powers, "Effectiveness of palivizumab: evaluation of outcomes from the 1998 to 1999 respiratory syncytial virus season. The Palivizumab Outcomes Study Group," The Pediatric Infectious Disease Journal, vol. 19, no. 11, pp. 1068-1071, 2000.

[34] Palivizumab Outcomes Registry Study Group, "Palivizumab prophylaxis of respiratory syncytial virus disease in 20002001: results from the palivizumab outcomes registry," Pediatric Pulmonology, vol. 35, no. 6, pp. 484-489, 2003.

[35] B. Paes, I. Mitchell, A. Li, and K. L. Lanctôt, "A comparative study of respiratory syncytial virus (RSV) prophylaxis in premature infants within the Canadian Registry of Palivizumab (CARESS)," European Journal of Clinical Microbiology \& Infectious Diseases, vol. 31, no. 10, pp. 2703-2711, 2012.

[36] S. G. Chang, M. S. Park, and J. E. Yu, "Outcomes of palivizumab prophylaxis for respiratory syncytial virus infection in preterm children with bronchopulmonary dysplasia at a single hospital in Korea from 2005 to 2009," Journal of Korean Medical Science, vol. 25, no. 2, pp. 251-256, 2010.

[37] P. M. A. Alexander, L. Eastaugh, J. Royle, A. J. Daley, L. S. Shekerdemian, and D. J. Penny, "Respiratory syncytial virus immunoprophylaxis in high-risk infants with heart disease," 
Journal of Paediatrics and Child Health, vol. 48, no. 5, pp. 395401, 2012.

[38] T. F. Feltes, A. K. Cabalka, H. C. Meissner et al., "Palivizumab prophylaxis reduces hospitalization due to respiratory syncytial virus in young children with hemodynamically significant congenital heart disease," The Journal of Pediatrics, vol. 143, no. 4, pp. 532-540, 2003.

[39] M. E. Speer, C. J. Fernandes, M. Boron, and J. R. Groothuis, "Use of palivizumab for prevention of hospitalization as a result of respiratory syncytial virus in infants with cystic fibrosis," The Pediatric Infectious Disease Journal, vol. 27, no. 6, pp. 559-561, 2008.

[40] A. G. Winterstein, E. Eworuke, D. Xu, and P. Schuler, "Palivizumab immunoprophylaxis effectiveness in children with cystic fibrosis," Pediatric Pulmonology, vol. 48, no. 9, pp. 874-884, 2013.

[41] B. Paes, I. Mitchell, H. Yi, A. Li, and K. L. Lanctôt, "Hospitalization for respiratory syncytial virus illness in down syndrome following prophylaxis with palivizumab," Pediatric Infectious Disease Journal, vol. 33, no. 2, pp. e29-e33, 2014.

[42] A. D. Chadha, W. Bao, J. Holloway, J. Mann, A. K. Rye, and D. E. Brown, "Respiratory syncytial virus morbidity and outpatient palivizumab dosing in South Carolina, 2004-2009," Southern Medical Journal, vol. 105, no. 8, pp. 399-404, 2012.

[43] B. Resch, W. Gusenleitner, W. D. Müller, and J. Haas, "Observational study of respiratory syncytial virus-associated hospitalizations and use of palivizumab in premature infants aged 29-32 weeks," European Journal of Clinical Microbiology \& Infectious Diseases, vol. 25, no. 2, pp. 120-122, 2006.

[44] M. Frogel, C. Nerwen, M. Boron et al., "Improved outcomes with home-based administration of palivizumab: results from the 2000-2004 palivizumab outcomes registry," The Pediatric Infectious Disease Journal, vol. 27, no. 10, pp. 870-873, 2008.

[45] J. P. T. Higgins and S. Green, Cochrane Handbook for Systematic Reviews of Interventions, Version 5.1.0, The Cochrane Collaboration, 2011.

[46] S. J. Clark, M. W. Beresford, N. V. Subhedar, and N. J. Shaw, "Respiratory syncytial virus infection in high risk infants and the potential impact of prophylaxis in a United Kingdom cohort," Archives of Disease in Childhood, vol. 83, no. 4, pp. 313316, 2000.

[47] "From the American Academy of Pediatrics: Policy statements-modified recommendations for use of Palivizumab for prevention of respiratory syncytial virus infections," Pediatrics, vol. 124, no. 6, pp. 1694-1701, 2009.

[48] J. A. Mullins, A. C. Lamonte, J. S. Bresee, and L. J. Anderson, "Substantial variability in community respiratory syncytial virus season timing," The Pediatric Infectious Disease Journal, vol. 22, no. 10, pp. 857-862, 2003.

[49] H. C. Meissner, L. J. Anderson, and L. K. Pickering, "Annual variation in respiratory syncytial virus season and decisions regarding immunoprophylaxis with palivizumab," Pediatrics, vol. 114, no. 4, pp. 1082-1084, 2004.

[50] S. H. Abman, J. W. Ogle, N. Butler-Simon, C. M. Rumack, and F. J. Accurso, "Role of respiratory syncytial virus in early hospitalizations for respiratory distress of young infants with cystic fibrosis," The Journal of Pediatrics, vol. 113, no. 5, pp. 826830, 1988 .

[51] D. Armstrong, K. Grimwood, J. B. Carlin et al., "Severe viral respiratory infections in infants with cystic fibrosis," Pediatric Pulmonology, vol. 26, no. 6, pp. 371-379, 1998. 


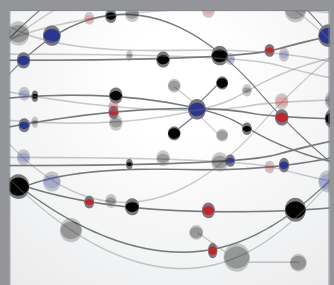

The Scientific World Journal
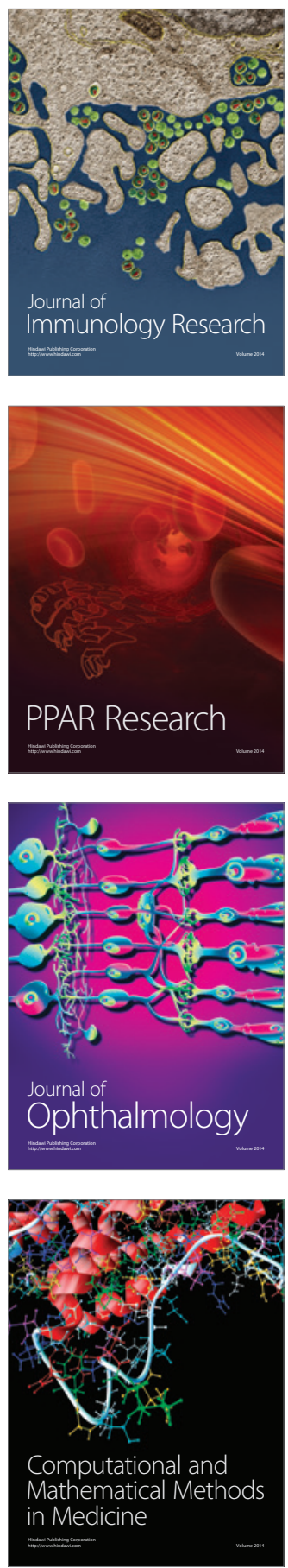

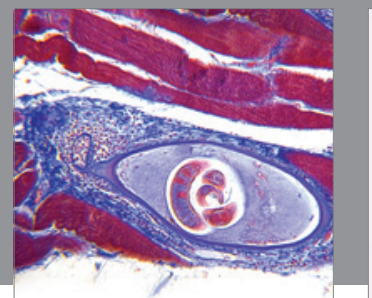

Gastroenterology

Research and Practice
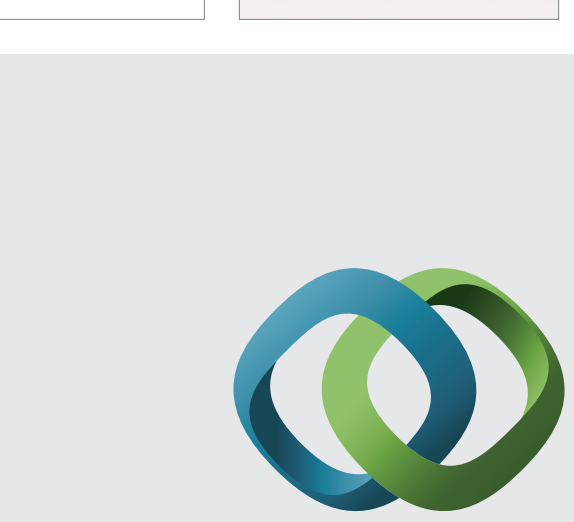

\section{Hindawi}

Submit your manuscripts at

http://www.hindawi.com
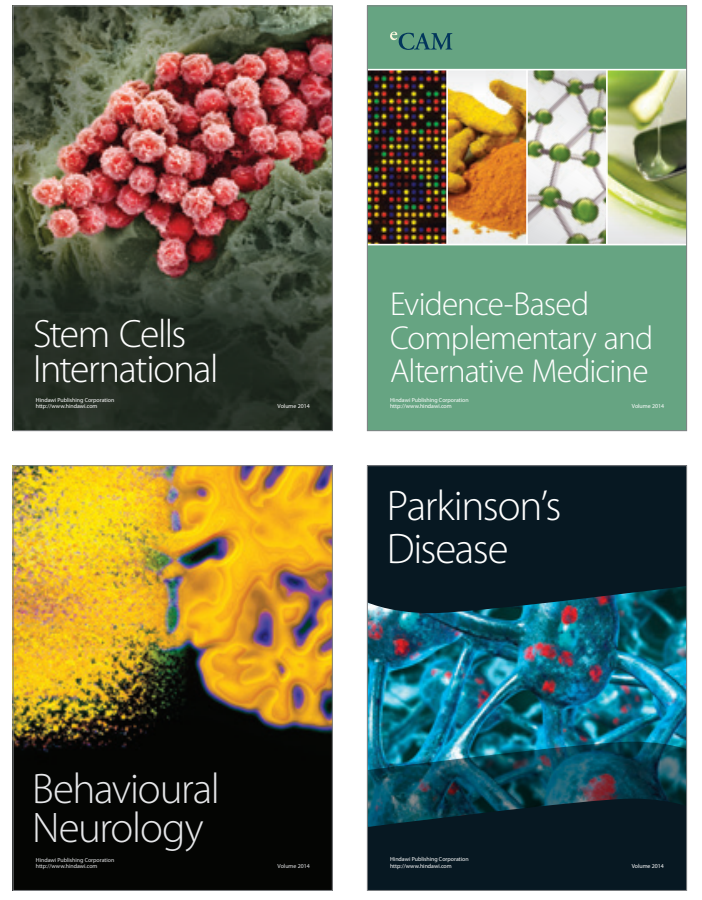
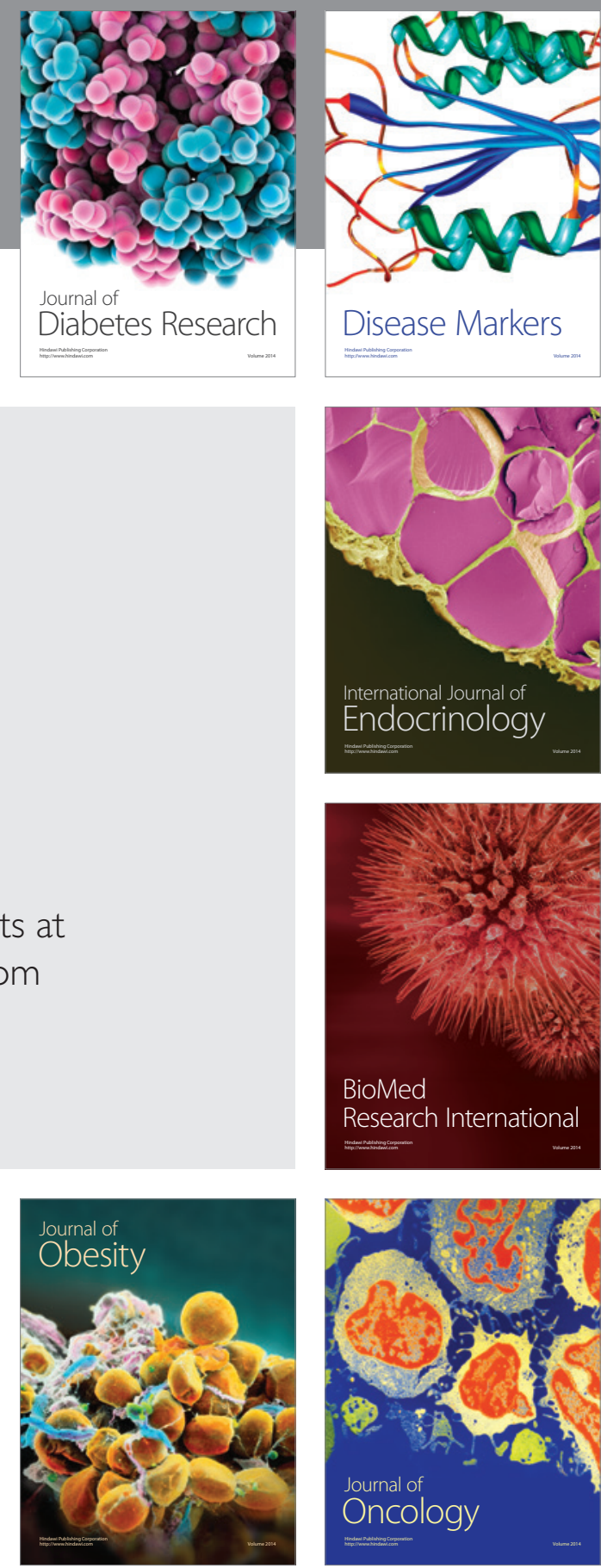

Disease Markers
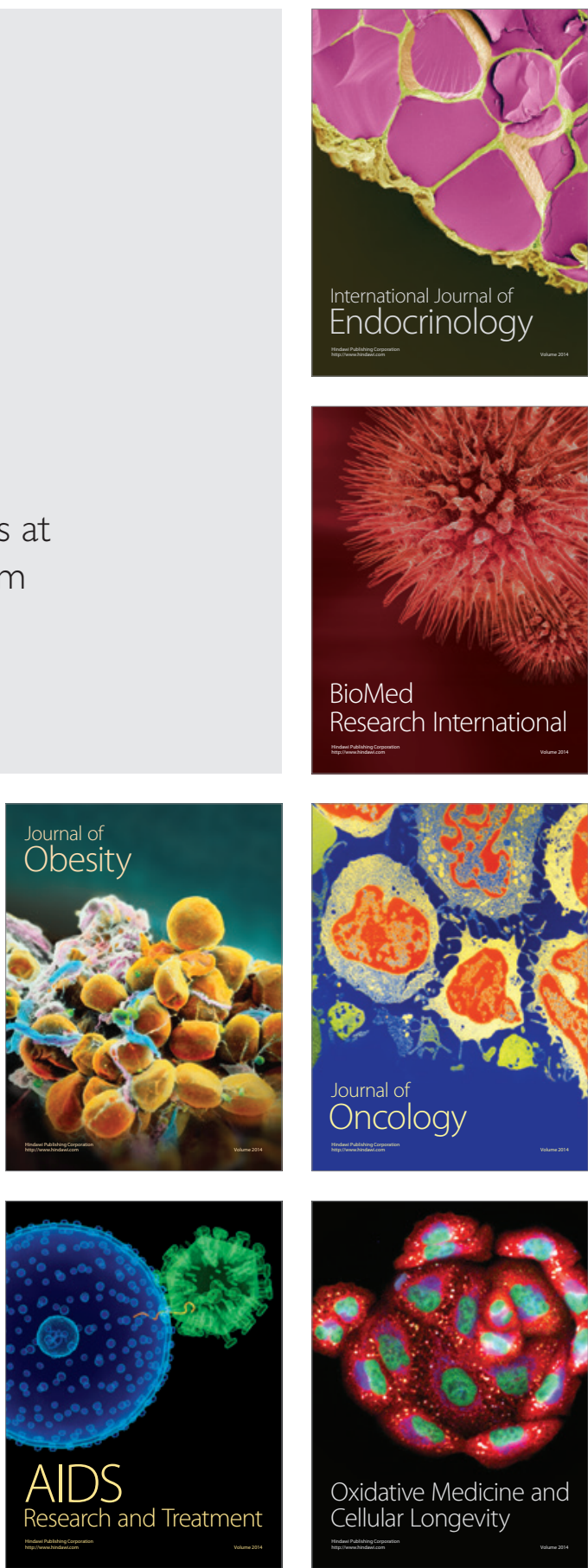